\title{
Successful Conversion From Thiazide to Tolvaptan in a Patient With Stage D Heart Failure and Chronic Kidney Disease Before Heart Transplantation
}

\author{
Teruhiko Imamura, ${ }^{1}$ MD, Koichiro Kinugawa, ${ }^{2}$ MD, Naoko Kato, ${ }^{1}$ PhD, Shun Minatsuki, ${ }^{1}$ MD, \\ Hironori Muraoka, ${ }^{1}$ MD, Toshiro Inaba, ${ }^{1}$ MD, Hisataka MaKI, ${ }^{1}$ MD, Taro Shiga, ${ }^{1}$ MD, \\ Masaru Hatano, ${ }^{1} \mathrm{MD}$, Atsushi Yao, ${ }^{1} \mathrm{MD}$, Shunei Kyo, ${ }^{2} \mathrm{MD}$, Minoru Ono, ${ }^{3} \mathrm{MD}$, \\ and Issei KomURo, ${ }^{1} \mathrm{MD}$
}

\begin{abstract}
SUMMARY
Chronic kidney disease (CKD) is often complicated with advanced heart failure because of not only renal congestion and decreased renal perfusion but also prolonged use of diuretics at higher doses, which sometimes results in hyponatremia. Preoperative CKD is known to be associated with poor prognosis after heart transplantation (HTx). We experienced a stage D heart failure patient with CKD and hyponatremia who was switched from trichlormethiazide to tolvaptan. His hyponatremia was normalized, and his renal function was improved after conversion to tolvaptan. In patients with stage D heart failure, it may be useful to administer tolvaptan with a concomitant reduction in the dose of diuretics in order to preserve renal function and avoid hyponatremia before HTx. (Int Heart J 2013; 54: 48-50)
\end{abstract}

Key words: Hyponatremia, Diuretics, Renal dysfunction

$\mathrm{C}$ hronic kidney disease (CKD) and hypervolemic hyponatremia are often complicated with advanced heart failure, and are not only attributable to renal congestion and reduced renal perfusion but also to extended use of diuretics at higher doses. ${ }^{1-5)}$ Heart transplantation (HTx) is the comprehensive solution for patients with stage D heart failure refractory to optimal medical therapy thus far, ${ }^{6}$ but it is well known that preoperative CKD results in a poor prognosis after HTx. ${ }^{7}$ It is especially important to preserve preoperative renal function since postoperative immunosuppressive therapy cannot help some kinds of renal impairment. ${ }^{8)}$ We experienced a stage D heart failure patient with CKD who was administered tolvaptan (TLV) and a vasopressin type 2 (V2) receptor antagonist, along with discontinuation of trichlormethiazide. His hyponatremia normalized and his renal function improved. Furthermore, his renal function remained within an acceptable range after HTx under immunosuppressive therapy including a calcineurin inhibitor.

\section{Case Report}

The patient was a 53-year-old male with ischemic cardiomyopathy (Figure 1, Table) who had received coronary artery bypass surgery in March 2010. His heart failure worsened even after the bypass surgery, and he eventually became dependent on intravenous infusion of inotropes regardless of maximum medical therapy including carvedilol, enalapril, spironolactone, trichlormethiazide, and furosemide in August 2010. He was transferred to our hospital as a possible candidate for HTx in September 2010. His laboratory data on admission showed $137 \mathrm{mEq} / \mathrm{L}$ of serum sodium, $1.9 \mathrm{mg} / \mathrm{dL}$ of serum total bilirubin, $1.2 \mathrm{mg} / \mathrm{dL}$ of serum creatinine, and $636 \mathrm{pg} / \mathrm{mL}$ of plasma B-type natriuretic peptide. Transthoracic echocardiography indicated an ejection fraction of $28 \%$ and left ventricular diastolic diameter of $67 \mathrm{~mm}$. A hemodynamic study performed in December 2010 demonstrated that mean right atrial pressure was $2 \mathrm{mmHg}$, mean pulmonary capillary wedge pressure was $21 \mathrm{mmHg}$, and the cardiac index was $2.2 \mathrm{~L} /$ minute $/ \mathrm{m}^{2}$ under continuous infusion of inotropes. He was then listed in February 2011 as a recipient of HTx. In the meantime, his serum creatinine increased to $1.4 \mathrm{mg} / \mathrm{dL}$ and his serum sodium concentration decreased below $130 \mathrm{mEq} / \mathrm{L}$ probably because of incremental dose of diuretics. However, we could not decrease the dose of diuretics for fear of inducing fatal decompensation.

In November 2011, he received furosemide at $60 \mathrm{mg} / \mathrm{day}$, torasemide at $4 \mathrm{mg} / \mathrm{day}$, and trichlormethiazide at $4 \mathrm{mg} / \mathrm{day}$ as diuretics. We then considered whether his renal dysfunction and hyponatremia could be reversed by reduction of any of the above diuretics. We chose to discontinue trichlormethiazide since $4 \mathrm{mg} /$ day of the thiazide might be excessive in combination with loop diuretics. However, we were concerned that the condition of his heart failure did not allow us simple cessation of trichlormethiazide, so we decided to introduce TLV at 3.75

From the Departments of ${ }^{1}$ Cardiovascular Medicine, ${ }^{2}$ Therapeutic Strategy for Heart Failure, and ${ }^{3}$ Cardiothoracic Surgery, Graduate School of Medicine, The University of Tokyo, Tokyo, Japan.

Address for correspondence: Koichiro Kinugawa, MD, Department of Therapeutic Strategy for Heart Failure, Graduate School of Medicine, The University of Tokyo, 7-3-1 Hongo, Bunkyo-ku, Tokyo 113-8655, Japan.

Received for publication October 11, 2012.

Revised and accepted November 29, 2012. 


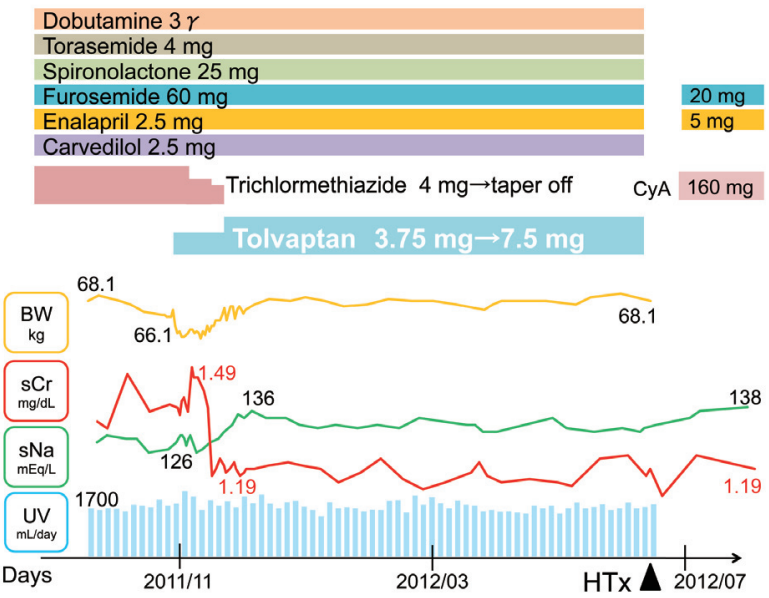

Figure 1. Clinical course of the case before and after the administration of tolvaptan. BW indicates body weight; sCr, serum creatinine; sNa, serum sodium; UV, urine volume; HTx, heart transplantation; and CyA, cyclosporin.

Table. Laboratory, Urine, and Echocardiographic Parameters

\begin{tabular}{lccc}
\hline & $\begin{array}{c}1 \text { month } \\
\text { before the } \\
\text { administra- } \\
\text { tion of TLV }\end{array}$ & $\begin{array}{c}1 \text { month } \\
\text { after the } \\
\text { administra- } \\
\text { tion of TLV }\end{array}$ & $\begin{array}{c}2 \text { months } \\
\text { after HTx }\end{array}$ \\
& & & \\
\hline Laboratory parameters & 7300 & 5600 & 7400 \\
\hline White blood cell count, & 11.2 & 10.7 & 11.1 \\
$\quad \times 10^{3} / \mu \mathrm{L}$ & 30.3 & 22.9 & 24.3 \\
Hemoglobin, g/dL & 128 & 136 & 138 \\
Platelets, $\times 10^{4} / \mu \mathrm{L}$ & 0.7 & 0.9 & 0.7 \\
Serum sodium, mEq/L & 18 & 15 & 8 \\
Serum total bilirubin, mg/dL & 16 & 12 & 14 \\
Serum AST, IU/L & 30.3 & 19.5 & 38.8 \\
Serum ALT, IU/L & 1.44 & 1.17 & 1.19 \\
Serum BUN, mg/dL & 432 & 330 & 100 \\
Serum creatinine, mg/dL & 262 & 279 & 284 \\
Serum BNP, pg/mL & & & \\
Serum osmolality, mOsm/L & 13 & 21 & 65 \\
Echocardiographic parameters & 62 & 66 & 48 \\
\hline Ejection fraction, \% & & & \\
LVDd, mm & & & \\
\hline
\end{tabular}

TLV indicates tolvaptan; HTx, heart transplantation; AST, aspartate amino transferase; ALT, alanine aminotransferase; BUN, blood urea nitrogen; BNP, B-type natriuretic peptide; and LVDd, left ventricular diastolic diameter.

$\mathrm{mg}$ /day and then titrated up to $7.5 \mathrm{mg} /$ day in place of the thiazide. After switching to TLV, serum creatinine decreased below $1.2 \mathrm{mg} / \mathrm{dL}$ and serum sodium normalized up to $135 \mathrm{mEq} /$ L. The urine osmolality responses after the administration of TLV were typical for a responder of this V2 receptor antagonist (Figure 2). He underwent HTx in July 2012, and his serum creatinine remained below $1.5 \mathrm{mg} / \mathrm{dL}$ regardless of cyclosporine treatment.

\section{Discussion}

Elevated venous pressure, low cardiac output, neurohormonal activation including the renin-angiotensin system con-

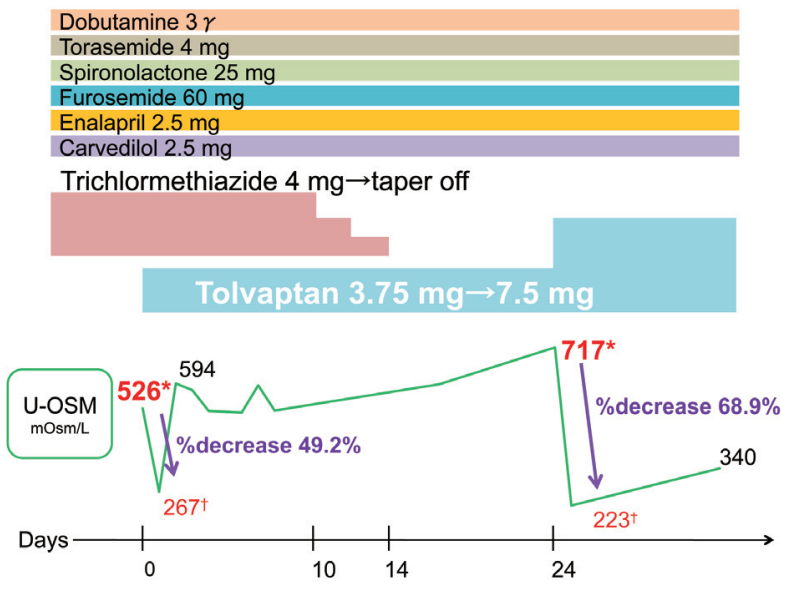

Figure 2. Time course of urine osmolality during the administration of tolvaptan. U-OSM indicates urine osmolality. " data were obtained just before the administration of tolvaptan (baseline urine osmolality). "Data were obtained 4 hours after the administration of tolvaptan.

tribute to renal dysfunction in patients with advanced heart failure. ${ }^{1)}$ Hypervolemic hyponatremia results from water retention by way of increases in arginine vasopressin (AVP) levels and/or decreases in glomerular filtration. ${ }^{2)}$ Especially in patients with stage D heart failure, almost all patients are dependent on high doses of diuretics for long periods, which facilitate both renal dysfunction and hyponatremia. Diuretic-induced renal dysfunction is due to decreased glomerular filtration by secondary stimulation of renin/angiotensin and norepinephrine secretion. On the other hand, hyponatremia caused by diuretics is attributable to increased excretion of sodium in urine and inappropriate AVP secretion against low or normal serum osmolality. ${ }^{3-5)}$ Consistently, our patient had a measurable concentration of AVP (2.7 pg/mL) despite low serum osmolality (262 $\mathrm{mOsm} / \mathrm{L}$ ) when he had hyponatremia just before conversion to TLV. Moreover, several investigators demonstrated that there was a dose-dependent association between diuretic use and mortality. ${ }^{9,10)}$ Even if we acknowledge such drawbacks of higher doses of diuretics, we could not avoid using considerable amounts of diuretics because he easily fell into a decompensated state without them.

Recently, TLV has been demonstrated to be advantageous in the correction of hyponatremia, ${ }^{11,12)}$ stabilization of hemodynamic state, ${ }^{13)}$ and amelioration of congestion without a worsening of renal function. ${ }^{14)}$ Administration of TLV along with discontinuation of trichlormethiazide successfully reversed his hyponatremia and renal dysfunction. We decided to discontinue trichlormethiazide because of its relatively weak diuretic effect and worsening effect on renal function compared with loop diuretics. ${ }^{5)}$ Several authors including us have reported that ventricular assist device (VAD) implantation can reverse endorgan dysfunction significantly. ${ }^{15,16)}$ Currently, approximately $90 \%$ of Japanese recipients require VAD support for bridge to $\mathrm{HTx}{ }^{7)}$ mainly because of the progressive decline in end-organ function that occurs in most patients with end-stage heart failure. Fortunately, he could be managed without VAD implantation until HTx, but preserving renal function was especially important considering the long waiting period before HTx. Moreover, treatment with a calcineurin inhibitor after HTx of- 
ten worsens renal function by decreasing the glomerular filtration ratio due to constriction of afferent glomerular arterioles of the kidneys, ${ }^{8)}$ but thanks to the preoperative recovery of renal function by conversion of diuretics, his serum creatinine level remained below $1.5 \mathrm{mg} / \mathrm{dL}$ after HTx.

We administered TLV at first at $3.75 \mathrm{mg} /$ day because of concern of a decrease of blood pressure due to large-scale diuresis. Urine osmolality was sufficiently higher than serum osmolality before TLV treatment, and significantly decreased at 4 hours after the administration of TLV, which implied effective excretion of free water. ${ }^{13)}$ The continuous decrease in urine osmolality after the titration of TLV up to $7.5 \mathrm{mg} /$ day indicated that TLV was sufficiently effective. Patients with more advanced CKD may not respond to TLV because of their inability to concentrate and dilute urine, which can be partly explained by an attenuated V2 receptor/aquaporin-2 system in the collecting duct, ${ }^{17)}$ though this has not been definitively determined. As we have previously reported, ${ }^{18)}$ responders to TLV are normally associated with significant decreases in urine osmolality after TLV treatment (ie, $>26 \%$ decrease of urine osmolality at 4-6 hours) from considerably high baseline levels (ie, > $352 \mathrm{mOsm} / \mathrm{L}$ ), which represent preserved function of the collecting duct including abilities to both concentrate and dilute urine. We were able to confirm in this case the criteria for TLV responders we proposed previously.

In conclusion, though no previous report has demonstrated an advantage in the conversion of conventional diuretics to TLV thus far, the present findings strongly suggest that TLV may be useful for improving hyponatremia and CKD, which may also lead to successful HTx in such cases waiting for long periods before HTx.

\section{REFERENCES}

1. Hillege HL, Girbes AR, de Kam PJ, et al. Renal function, neurohormonal activation, and survival in patients with chronic heart failure. Circulation 2000; 102: 203-10.

2. Douglas I. Hyponatremia: why it matters, how it presents, how we can manage it. Cleve Clin J Med 2006; 73 Suppl 3: S4-12. (Review)

3. Leier CV, Dei Cas L, Metra M. Clinical relevance and management of the major electrolyte abnormalities in congestive heart failure: hyponatremia, hypokalemia, and hypomagnesemia. Am Heart J 1994; 128: 564-74. (Review)

4. Abdel-Qadir HM, Tu JV, Yun L, Austin PC, Newton GE, Lee DS. Diuretic dose and long-term outcomes in elderly patients with heart failure after hospitalization. Am Heart J 2010; 160: 264-71. el.
5. de Silva R, Nikitin NP, Witte KK, et al. Incidence of renal dysfunction over 6 months in patients with chronic heart failure due to left ventricular systolic dysfunction: contributing factors and relationship to prognosis. Eur Heart J 2006; 27: 569-81.

6. Hunt SA, Abraham WT, Chin MH, et al. ACC/AHA 2005 Guideline Update for the Diagnosis and Management of Chronic Heart Failure in the Adult: a report of the American College of Cardiology/American Heart Association Task Force on Practice Guidelines (Writing Committee to Update the 2001 Guidelines for the Evaluation and Management of Heart Failure): developed in collaboration with the American College of Chest Physicians and the International Society for Heart and Lung Transplantation: endorsed by the Heart Rhythm Society. Circulation 2005; 112: e154-235.

7. Stehlik J, Edwards LB, Kucheryavaya AY, et al. The Registry of the International Society for Heart and Lung Transplantation: Twenty-eighth Adult Heart Transplant Report--2011. J Heart Lung Transplant 2011; 30: 1078-94.

8. Baran DA, Galin ID, Gass AL. Calcineurin inhibitor-associated early renal insufficiency in cardiac transplant recipients: risk factors and strategies for prevention and treatment. Am J Cardiovasc Drugs 2004; 4: 21-9. (Review)

9. Eshaghian S, Horwich TB, Fonarow GC. Relation of loop diuretic dose to mortality in advanced heart failure. Am J Cardiol 2006; 97: 1759-64.

10. Hasselblad V, Gattis Stough W, Shah MR, et al. Relation between dose of loop diuretics and outcomes in a heart failure population: results of the ESCAPE trial. Eur J Heart Fail 2007; 9: 1064-9.

11. Schrier RW, Gross P, Gheorghiade M, et al. Tolvaptan, a selective oral vasopressin V2-receptor antagonist, for hyponatremia. N Engl J Med 2006; 355: 2099-112.

12. Imamura $\mathrm{T}$, Kinugawa $\mathrm{K}$, Shiga $\mathrm{T}$, et al. Correction of hyponatremia by tolvaptan before left ventricular assist device implantation. Int Heart J 2012; 53: 391-3.

13. Udelson JE, Orlandi C, Ouyang J, et al. Acute hemodynamic effects of tolvaptan, a vasopressin V2 receptor blocker, in patients with symptomatic heart failure and systolic dysfunction: an international, multicenter, randomized, placebo-controlled trial. Am Coll Cardiol 2008; 52: 1540-5.

14. Konstam MA, Gheorghiade M, Burnett JC Jr, et al. Effects of oral tolvaptan in patients hospitalized for worsening heart failure: the EVEREST Outcome Trial. JAMA 2007; 297: 1319-31.

15. Singh M, Shullo M, Kormos RL, et al. Impact of renal function before mechanical circulatory support on posttransplant renal outcomes. Ann Thorac Surg 2011; 91: 1348-54.

16. Imamura T, Kinugawa K, Shiga T, et al. Preoperative levels of bilirubin or creatinine adjusted by age can predict their reversibility after implantation of left ventricular assist device. Circ J. (in press)

17. Kwon TH, Frøkiaer J, Knepper MA, Nielsen S. Reduced AQP1, -2 , and -3 levels in kidneys of rats with CRF induced by surgical reduction in renal mass. Am J Physiol 1998; 275: F724-41.

18. Imamura T, Kinugawa K, Shiga T, et al. Novel criteria with urine osmolality effectively predict responses to tolvaptan in decompensated heart failure patients - An association between non-responders and chronic kidney disease. Circ J. (in press) 